\title{
超交联微孔聚合物研究进展
}

\author{
谭良骁＼cjkstart谭必恩* \\ (华中科技大学 化学与化工学院 武汉 430074)
}

\begin{abstract}
摘要 超交联微孔聚合物是一类重要的多孔聚合物材料，由于其具有高比表面积、合成条件温和、单体来源广泛等优 点而成为研究的热点. 根据不同阶段合成方法的差异, 超交联聚合物主要由以下三种方法制备得到: (1)含官能团聚合 物前体的后交联; (2)功能化小分子单体的一步法自缩聚; (3)通过外交联剂 “编织” 刚性的芳香族单体. 本文介绍了超交 联聚合物的发展过程，着重对三种合成超交联聚合物的方法、同时对微孔聚合物微观形貌的控制以及其在气体储存、 分离、催化等方面的应用进行了总结. 最后提出了超交联聚合物的缺陷和所面临的挑战, 并对未来超交联微孔聚合物 的发展前景进行了展望，指明了超交联聚合物发展的新方向.
\end{abstract}

关键词 微孔; 超交联聚合物; 后交联; 自缩合; 外交联

\section{Research Progress in Hypercrosslinked Microporous Organic Polymers}

Tan, Liangxiao Tan, Bien*

(School of Chemistry and Chemical Engineering, Huazhong University of Science and Technology, Wuhan 430074)

\begin{abstract}
Hypercrosslinked microporous polymers are currently an important class of porous polymer materials and receiving great interest due to their advantages such as high surface area, moderate synthetic conditions and diverse building blocks. According to the difference between the synthetic methods, hypercrosslinked polymers are mainly prepared by the following three strategies: polymer precursor post-crosslinking, one-step self-polycondensation of multifunctional monomers and external crosslinker knitting rigid aromatic compounds. In this review, we introduce the development of hypercrosslinked polymer in detail as well as investigate various synthetic methods and polymer networks with controlled micro-morphology, the broad practical and potential applications including gas storage, adsorption, separation and heterogeneous catalysis were also discussed. In the end, we talk about the disadvantage of hypercrosslinked polymers and challenges in the future as well as predict the further development on synthesis and application.
\end{abstract}

Keywords microporous; hypercrosslinked polymers; post-crosslinking; self-polycondensation; external crosslinking

\section{1 引言}

根据国际纯粹与应用化学联合会(IUPAC)的分类, 孔径尺寸小于 $2 \mathrm{~nm}$ 的孔被称为微孔 ${ }^{[1]}$, 而富含微孔的材 料被统称为微孔材料. 微孔材料由于其独特的孔结构性 质, 在气体储存、分离、多相催化、传感器、储能等应 用领域引起了人们的广泛关注 ${ }^{[2]}$. 近几十年来，随着对 材料性能要求的提高, 传统的多孔材料如活性炭 ${ }^{[3,4]}$ 、沸 石 ${ }^{[5]}$ 、介孔硅 ${ }^{[6]}$ 等已经不能满足日益发展的能源环境需 求, 对多孔材料研究的重心渐渐偏移到新型微孔材料的 开发上来. 有机微孔聚合物材料(microporous organic polymers, MOPs $)^{[7]}$ 是一类由较轻的 $\mathrm{C} 、 \mathrm{H} 、 \mathrm{O} 、 \mathrm{~N}$ 等元素 组成的新型微孔材料, 具有低的骨架密度、较高的比表 面积、良好的物理化学稳定性等优点, 相比于其它一些 微孔材料, MOPs 更大的优势是可以通过改变构建单元 的官能团或者采用不同的合成方法来制备多功能化的
产物, 从而实现 MOPs 功能化的目的 ${ }^{[8,9]}$. 而且, 聚合物 合成已经发展成为工业化的技术，商业化聚合物材料的 大规模生产也有了许多实例 ${ }^{[10]}$. 因而 MOPs 逐渐成为了 一种有极大发展潜力和广泛应用前景的新型孔材料, 成 为了研究的热点.

通常, MOPs 是通过一些能够形成分子间 $\mathrm{C}-\mathrm{C}$ 单键 的反应得到的 ${ }^{[7]}$. 根据它们的结构特点以及合成方式的 不同, 主要将 MOPs 分为以下四类: 共轭微孔聚合物 (conjugated microporous polymers, CMPs) ${ }^{[1,12]}$, 自具微 孔聚合物(polymers of intrinsic microporosity, PIMs) ${ }^{[13]}$, 共价有机网络(covalent organic frameworks, $\mathrm{COFs})^{[14]}$ 以 及超交联聚合物(hypercrosslinked polymers, HCPs) ${ }^{[15 \sim 17]}$.

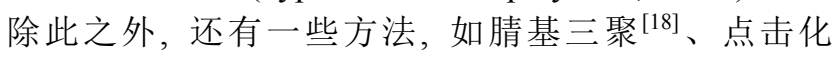
学 ${ }^{[19,20]}$ 和氧化聚合 ${ }^{[21,22]}$ 等反应能够用于 MOPs 的合成. 虽然 MOPs 的合成方法多种多样, 然而 CMPs、多芳香 骨架(PAFs) 以及其它一些 MOPs 的合成需要用到复杂的

* E-mail: bien.tan@mail.hust.edu.cn; Tel.: 027-87558172; Fax: 027-87543632

Received February 3, 2015; published March 16, 2015.

Project supported by the National Natural Science Foundation of China (Nos. 21474033, 51273074, 51173058) and the Program for New Century Excellent Talents in University (No. NCET-10-0389).

项目受国家自然科学基金(Nos. 21474033, 51273074, 51173058)和新世纪优秀人才计划(No. NCET-10-0389)资助. 
单体单元, 昂贵的过渡金属催化剂, 或者需要在较苛刻 的条件下进行 ${ }^{[7]}$, 因此 MOPs 的低成本制备仍然是一个 难以解决的问题.

超交联聚合物材料是一类基于付-克烷基化反应 ${ }^{[23]}$ 制备得到的多孔材料 ${ }^{[24]}$. 超交联聚合物的发现借鉴了 其它材料的合成中所使用 “交联” 的概念, 而在超交联 聚合物的制备过程中, 交联的程度更大, 所得到的聚合 物网络呈现出高度刚性, 阻止了聚合物链的紧密收缩, 因而在分子链间存在一些空隙形成了孔 ${ }^{[15]}$. 由于交联 网络的高度刚性, 超交联微孔聚合物材料一般具有稳定 的孔结构, 较高的比表面积和较大的微孔体积. 经过数 十年的发展, 随着不同结构性质单体的开发应用, 超交 联微孔聚合物材料的种类越来越多, 聚合物的性能有了 显著的提高，应用范围也有了极大的扩展. 根据不同阶 段合成方法的差异, 超交联聚合物主要由以下三种方法 制备得到: (1)含官能团聚合物前体的后交联; (2)功能化 小分子单体一步法自缩聚; (3)通过外交联剂 “编织” 刚 性的芳香族单体. 本文将介绍超交联聚合物的发展过 程, 着重对三种合成超交联聚合物的方法、同时对微孔 聚合物微观形貌的控制以及其在气体储存、分离、催化 等方面的应用进行总结.

\section{2 聚合物前体后交联法}

超交联聚苯乙烯网络(Davankov 树脂)由 Davankov 在 1971 年报道 ${ }^{[23]}$, 被认为是最早的超交联聚合物, 通 过可溶的线性聚苯乙烯聚合物或溶胀的凝胶型聚苯乙 烯-二乙烯基苯共聚物的深度交联得到 ${ }^{[25]}$. 反应机理如 图 1 所示, 首先, 溶解过程伴随着聚合物分子链的解离 以及溶剂分子的扩散, 分子链间的空间被溶剂分子填 充. 之后, 交联反应在溶剂分子周围发生, 聚合物分子 链以一种分散开的状态被交联固定. 反应结束后, 溶剂 分子被除去, 分子链之间的空隙就形成了孔 ${ }^{[26]}$. 交联反 应的程度越高, 所形成聚合物分子链的刚性越强, 形成 孔的结构就越稳定, 孔尺寸也会随着交联度的提高而降 低.

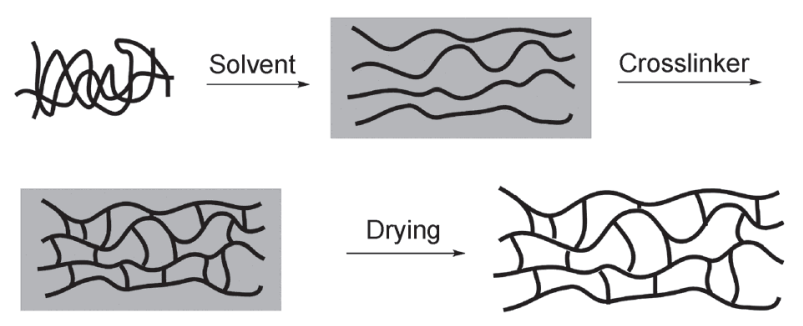

图 1 溶胀聚苯乙烯的超交联过程 ${ }^{[26]}$

Figure 1 The hypercrosslinking process of swollen polystyrene ${ }^{[26]}$

随后，在无水 $\mathrm{SnCl}_{4}$ 的存在下，Davankov 等 ${ }^{[27]}$ 使用 氯甲基甲醚作为交联剂，通过交联线性的聚苯乙烯制备 了分子内超交联的可溶性微孔材料. 这类微孔聚合物的 比表面积可达到 $680 \sim 1000 \mathrm{~m}^{2} / \mathrm{g}$, 在色谱分析、液相分
离以及有机蒸汽的吸附方面显示了较好的应用潜能. 相 比于氯甲基甲醚，四氯化碳是一种更好的低毒性交联 剂. Hradil 等 ${ }^{[28]}$ 报道了四氯化碳交联的聚苯乙烯类树脂. 在无水 $\mathrm{AlCl}_{3}$ 或 $\mathrm{FeCl}_{3}$ 的催化下，四氯化碳发生付-克烷 基化反应形成了苯环间的- $\mathrm{CCl}_{2}$-桥连，之后通过水解形 成了-CO-桥连结构. 通过这种桥连得到的聚合物网络比 表面积能够达到 $1000 \mathrm{~m}^{2} / \mathrm{g}$ 以上.

作为聚苯乙烯类似物，二乙烯基苯一氯甲基苯乙烯 共聚物(DVB-VBC)也是一种后交联反应的有效前体. 在路易斯酸的催化下，聚合物分子链上的氯甲基基团与 相邻的苯环发生付-克烷基化反应，脱去了分子末端的 氯原子并与相邻苯环连接, 形成了一种高度交联网络结 构 $^{[29]}$. 因此, 由这种前体交联得到的超交联聚合物通常 具有更强的刚性, 得到的微孔聚合物比表面积更高, 交 联网络中一般只含有微孔结构. Sherrington 等 ${ }^{[30]}$ 通过 DVB-VBC 前体制备得到了一系列的超交联聚合物(图 2 ), 并研究了不同的反应条件，例如：氯甲基苯乙烯的 单体比例，反应溶剂和催化剂等对孔结构和聚合物比表 面积的影响. 根据不同的反应条件，超交联聚合物比表 面积为 $300 \sim 2000 \mathrm{~m}^{2} / \mathrm{g}$ 不等. 使用摩尔分数 $0.02 x$ 的 DVB-VBC 前体，当交联时间为 $15 \mathrm{~min}$ 时，得到聚合物 比表面积只有 $1200 \mathrm{~m}^{2} / \mathrm{g}$ ，而延长交联时间至 $18 \mathrm{~h}$ ，比表 面积达到最大值 $2090 \mathrm{~m}^{2} / \mathrm{g}$.

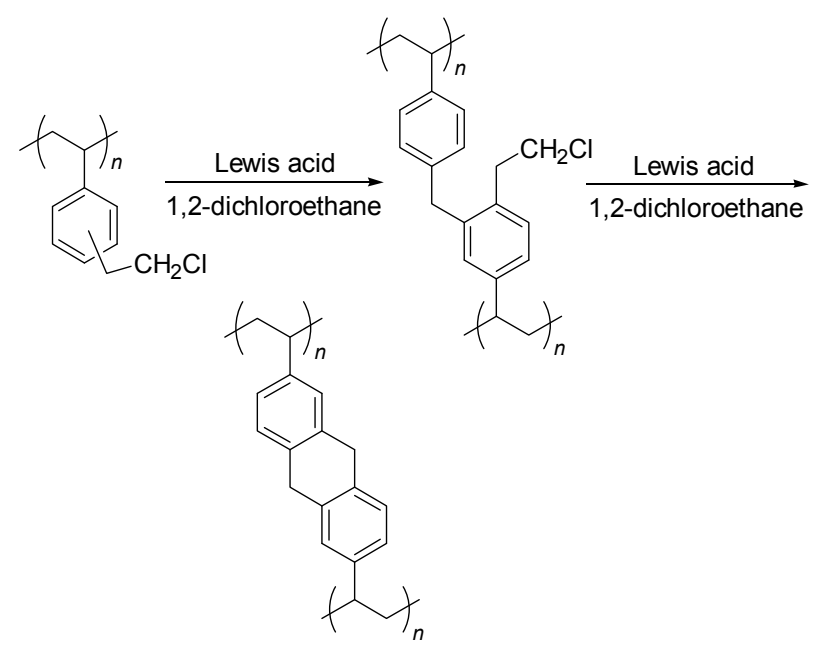

图 2 二乙烯基苯-氯甲基苯乙烯树脂的后交联 ${ }^{[30]}$

Figure 2 The post-crosslinking of DVB-VBC precursor resins ${ }^{[30]}$

目前, 对聚苯乙烯类超交联聚合物的合成研究已经 比较深入，但是关于具有可控孔径尺寸和孔分布超交联 聚合物的报道还很少. Tan 小组 ${ }^{[31}$ 选择典型的 DVBVBC 共聚物作为反应前体, 通过改变交联剂 DVB 的含 量, 有效地调控了 Davankov 树脂的孔径及孔径分布, 得到了窄分布的纯微孔结构. 实验结果表明, 通过增加 前体中 DVB 的含量, 超交联微孔聚合物 HCP-DVB$\mathrm{VBC}$ 的孔结构能够被精确地从大孔向微孔调控. 氮气 吸附测试结果表明, 随着 DVB 含量的增加, HCPDVB-VBC 的 BET 比表面积先上升然后下降. 当 DVB 
含量为 $0.02 w$ 时, HCP-DVB-VBC 的 BET 比表面积最高, 达到 $2064 \mathrm{~m}^{2} / \mathrm{g}$. DVB 含量从 $0.01 w$ 增加到 $0.1 w$ 过程中, 超交联产物孔径尺寸逐渐减小, 微孔结构更加均一, 孔 径分布更加狭窄. 当DVB含量超过 $0.07 w$ 时, 微孔聚合 物的氮气吸附解吸附曲线呈现出典型的 I 型曲线特征, 表明形成了纯微孔的聚合物网络. 根据实验结果, 对超 交联聚合物比表面积和孔径分布变化趋势进行分析, 提 出了如下的反应机理. 对于不含 DVB 的聚合物前体, 分子链在溶剂中能够完全溶解, 呈现出一种无序的状 态, 苯环上的氯甲基官能团更容易与相隔较远分子链片 段的苯环接触. 交联反应发生时, 较远苯环连接形成了 较大的空隙, 最终发展为大孔结构. 含 DVB 的聚合物 前体受到 DVB 的初步交联作用, 在溶剂中分子链的运 动受到限制不能自由的分散在整个溶剂中. 当 DVB 含 量较低时, 初步交联程度较低, 分子链疏松, 交联反应 与不含 DVB 的前体相似, 得到的孔结构仍然不均一. 当 DVB 含量较高时, 由于 DVB 的初步交联固定作用, 分子链只能处于伸展状态, 苯环只能与邻近的苯环交 联, 最终得到了一种均匀的交联网络, 相比之下孔结构 更加均一. 因此, DVB 含量为 $0.02 \sim 0.1 w$ 的 HCP-DVBVBC 呈现出更加平滑、狭窄、均一的孔径分布. 微孔聚 合物孔径差异对聚合物性能的影响可以通过不同的气 体吸附结果来反映. 例如, $0.02 w$ DVB 含量的 HCPDVB-VBC 显示了最高的氮气吸附量, 但是 $0.05 w$ DVB 含量的 HCP-DVB-VBC 却有最高的氢气吸附量, 而 0.1 $w$ DVB 含量的 HCP-DVB-VBC 能吸附最大量的二氧化 碳. 这样完全不同的气体吸附结果也表明了更小的微孔 孔径和更高的微孔体积对小气体分子氢气和二氧化碳 的吸附是有益的.

为了对上述超交联聚合物中的孔结构的形成机理 有更深入的了解, Abbott等 ${ }^{[32]}$ 通过 Polymatic 软件模拟了 一系列苯乙烯-氯甲基苯乙烯超交联聚合物的交联反应 过程, 模拟包括溶胀状态的交联过程和从溶胀状态到干 态过程孔结构的变化. 结果表明, 超交联聚合物中极高 的孔隙率是聚合物在低密度下的高度交联得到的, 说明 实验开始阶段交联的作用至关重要. 通过模拟 VBC 的 含量对超交联聚合物比表面积的影响, 发现比表面积的 变化趋势与 Tan 报道的实验结果 ${ }^{[31]}$ 相一致. 通过对虚拟 合成中比表面积和孔径分布的模拟, 发现聚合物在溶胀 状态下的孔是逐渐形成的. 交联作用能够对聚合物分子 链进行重新分配, 随着交联时间的延长, 聚合物交联度 越来越大. 最后当反应结束, 产物从溶胀状态向干态变 化时, 聚合物的高度交联阻止了孔道的坉塌, 使得产物 具有较高的比表面积和较大的孔体积. 作为实验研究的 补充, 模拟方法对超交联聚合物孔结构形成提供了一种 更好的理解方式.

除聚苯乙烯类 DVB-VBC 共聚物外, 最近一些研究 表明含聚苯乙烯的嵌段聚合物在设计微孔聚合物结构
中也表现出较为明显的优势 ${ }^{[33,34]}$. Wu 小组 ${ }^{[34]}$ 使用甲基 丙烯酸甲酯一苯乙烯嵌段共聚物作为前体, 通过聚合物 自组装形成了一种具有核壳结构的纳米微球, 聚苯乙烯 壳层经四氯化碳交联后具有更强的刚性，能够在后续的 碳化过程中保持形貌, 得到的具有多级孔结构的碳纳米 中空微球, 在二氧化碳捕获、超级电容器等能源环境方 面有良好的应用前景.

\section{3 功能化小分子自缩聚法}

近些年来, Davamkov 树脂在合成方面有了较快的 发展，然而这种后交联方法的局限性限制了其实际应 用 ${ }^{[35]}$. 原因在于 Davankov 树脂的合成中必须先得到聚 合物前体, 然后超交联形成微孔网络, 极大限制了可选 择的单体种类以及超交联聚合物的性能. 那么, 超交联 聚合物是否可以直接通过含有功能基团的小分子单 体 ${ }^{[36]}$ 通过一步法聚合反应直接得到?

这方面的研究最早是由 Cooper 小组 ${ }^{[17]}$ 开展, 他们 选取了三种含氯甲基的芳环单体(图 3)：对二氯二甲苯 (DCX)、4,4'-二氯甲基-1,1'-联苯(BCMBP)和二氯甲基葱 (BCMA). 在不同路易斯酸的催化下, 通过改变三种不 同单体间的比例, 得到了一系列结构相似的微孔聚合物 网络. 氮气吸附解吸附表明了这些材料具有永久的微孔 结构, 最高 BET 比表面积可达 $1904 \mathrm{~m}^{2} / \mathrm{g}$, 与 Davankov 树脂比表面积相当. 随后发现基于 BCMCP 和 DCX 的微 孔聚合物在 $77 \mathrm{~K} / 15$ bar 条件下的氢气吸附高达 0.0368 $w$, 表明了这类微孔聚合物材料在氢气储存方面存在巨 大的潜力.
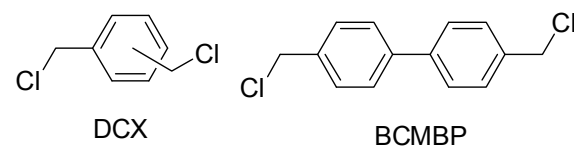

BCMBP

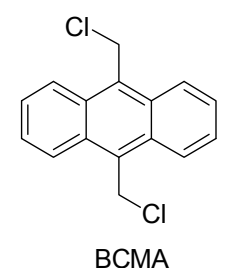

图 3 自缩合聚合反应的单体 ${ }^{[17]}$

Figure 3 Monomers used in self-polycondensation strategy ${ }^{[17]}$

天然气作为一种化石能源, 现在仍然在人类社会活 动中占据着重要的地位. 作为可以替代汽油等石油工业 产物的一种新型汽车燃料, 甲烷(天然气主要成分)的储 存也渐渐成为研究的热点. Cooper 等 ${ }^{[37]}$ 进一步研究了这 类自缩聚超交联聚合物对甲烷气体的吸附性能. 当 DCX 和 BCMCP 单体比例为 $1: 3$ 时, 得到的超交联微 孔聚合物在 $298 \mathrm{~K} / 20 \mathrm{bar}$ 条件下的甲烷吸附量能够达到 $5.4 \mathrm{mmol} / \mathrm{g}$, 表现出极好的应用前景. 更重要的是, 这 种合成方法能够制备出微孔聚合物的整块材料, 相比于 一般粉末颗粒材料更容易储存.

近些年来, 碳的捕获与封存技术 $(\mathrm{CCS})$ 被发现是现 阶段一种解决二氧化碳过量排放的有效方法, 而这种方 法的关键技术在于发明一种能够高效吸附二氧化碳的 
材料 ${ }^{[38]}$. Martin 等 ${ }^{[39]}$ 测试了这类微孔材料分别在大气压 和高压下的二氧化碳吸附能力. 测试结果表明材料的二 氧化碳吸附量与超交联微孔聚合物的孔结构性质相关, 在 $30 \mathrm{bar}$ 的高压下, 聚合物显示了 $13.4 \mathrm{mmol} / \mathrm{g}$ 的极高 二氧化碳吸附量. 而且由于较低的吸附热和良好的选择 性, 这类材料能够作为燃烧后二氧化碳捕获的高效材 料.

除了高比表面积, 很多研究表明了在聚合物网络中 引入功能基团如: 羧酸和胺基团能有效的提高二氧化碳 吸附量和二氧化碳对氮气的吸附选择性 ${ }^{[40 ~ 42]}$.

Yang 等 ${ }^{[43]}$ 将含氮单体三苯胺通过与 DCX 共聚引入 到超交联聚合物网络中. 随着单体 DCX 含量的提高, 得到聚合物的 BET 比表面积也从 318 增加到 $1530 \mathrm{~m}^{2} / \mathrm{g}$. 与猜想结果一致, 这种含氮聚合物网络对二氧化碳的吸 附量有了一定提高, 最高可达到 $4.6 \mathrm{mmol} / \mathrm{g}$. 随后, Yang 等 ${ }^{[44]}$ 研究了 BCMBP 与三苯胺共聚聚合物的烃类/ 水分离性能. 通过调整单体间的不同比例, 得到了一系 列高比表面积和含有永久微孔结构的超交联聚合物. 其 中, PBP-N-25 和 PBP-N-50 两种交联产物的比表面积分 别高达 1362 和 $1338 \mathrm{~m}^{2} / \mathrm{g}$, 它们苯/水蒸汽的分离选择性 分别为 53.5 和 63.6. 而且, 通过这两种单体的共聚能够 制备出 BET 比表面积为 $551 \mathrm{~m}^{2} / \mathrm{g}$ 的聚合物整块材料. 整 块聚合物具有良好的疏水性和低密度, 因而能够被应用 于在海洋中清洁泄漏的原油.

除了高比表面积的有机超交联微孔聚合物的合成, Chaikittisilp 等 ${ }^{[45]}$ 通过含有苄基氯末端功能团的立方硅 氧烷笼子的自缩聚反应制备得到了一种新型的多级微 孔一介孔超交联硅氧烷有机复合材料. 虽然合成过程中
伴随着硅氧烷笼子的坍塌, 但是苄基氯末端官能团能够 充当分子间连接的桥梁有效地构建超交联的网络，最后 得到的多级孔复合材料的 BET 比表面积和孔体积分别 达到 $2500 \mathrm{~m}^{2} / \mathrm{g} 、 3.3 \mathrm{~cm}^{3} / \mathrm{g}$. 这项研究表明了这种分子缩 聚形成超交联聚合物的方法同样能够被应用于有机无 机复合材料的制备.

之后, $\mathrm{Wu}$ 等 ${ }^{[46]}$ 使用类似的单体通过无水 $\mathrm{AlCl}_{3}$ 催化 的付一克烷基化反应同样制备得到了硅氧烷有机复合材 料. 与之前研究不同的是, 他们选择了含双键末端基团 的单体与苯单体共聚，最终得到了富含微孔一介孔的复 合材料. 最高 BET 比表面积为 $904 \mathrm{~m}^{2} / \mathrm{g}$, 最大孔体积为 $0.99 \mathrm{~cm}^{3} / \mathrm{g}$.

$\mathrm{Wu}$ 小组 ${ }^{[47]}$ 以苯基功能化的笼型聚倍半硅氧烷(八 苯基-POSS)作为反应单体, 在无水 $\mathrm{AlCl}_{3}$ 的催化下, 通 过四氯化碳交联制备了一种有机一无机纳米颗粒复合材 料. 经碳化、刻蚀后, 所得碳材料具有高达 $2264 \mathrm{~m}^{2} / \mathrm{g}$ 的 比表面积以及极高的微孔孔隙率，在二氧化碳捕获分离 以及有机小分子吸附等方面显示出良好的性能.

除了传统的含有氯甲基基团的单体能够自缩聚反 应, Tan 小组 ${ }^{[48]}$ 证明了含羟甲基官能团的茮醇类单体如: 对苯二甲醇(BDM)和苯甲醇(BA)也能够通过付一克烷基 化反应自缩聚形成具有高比表面积的超交联微孔聚合 物(图 4). 氮气吸附结果表明, 两种聚合物都有永久的微 孔结构, 比表面积分别高达 $847 \mathrm{~m}^{2} / \mathrm{g}$ (HCP-BDM) 和 $742 \mathrm{~m}^{2} / \mathrm{g}$ (HCP-BA). 这一结果突破了超交联反应单体 必须含有多个反应基团的传统认知, 将可成孔的自缩合 单体扩展到单官能团芳香族化合物，扩展了合成超交联 微孔聚合物材料可选单体范围.

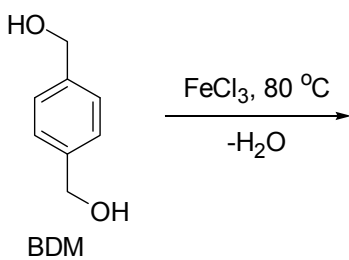

BDM

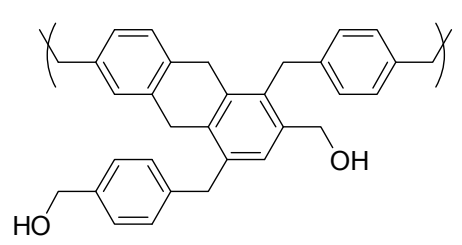

HCP-BDM

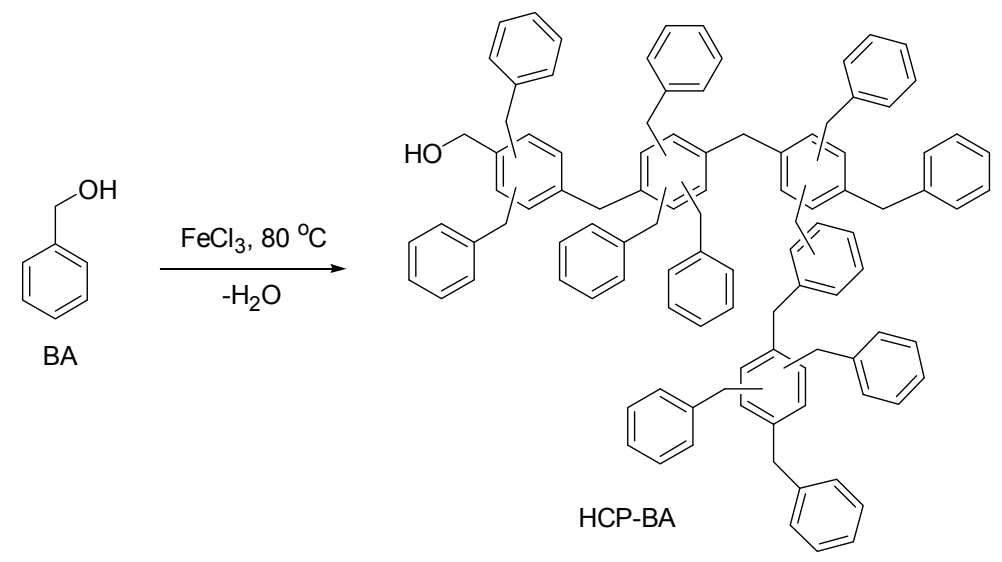

图 4 超交联苯二甲醇和超交联苯甲醇的合成 ${ }^{[48]}$

Figure 4 Synthesis of HCP-BDM and HCP-BA by Friedel-Crafts catalyzed self-polycondensation ${ }^{[48]}$ 
最近, Tan 小组 ${ }^{[49]}$ 的研究进一步扩展到不含官能团 单体的一步法自缩聚反应(图 5). 这种合成方法是基于 Scholl 偶联反应 ${ }^{[50,51]}$, 在 $\mathrm{AlCl}_{3}$ 催化剂的作用下, 两个相 邻苯环上的氢原子被消除, 同时形成一个新的 $\mathrm{C}-\mathrm{C}$ 单 键. 相比于传统的超交联聚合物, 这种由 Scholl 偶联方 法制备的微孔聚合物材料有明显的不同: 由于反应中不 需要发生交联的基团作为单体间的桥连单元, 芳香族单 体的共轭结构能够很好的保留, 得到的聚合物网络具有 整体的共轭结构. 与共轭微孔聚合物的制备相比, 这种 方法具有显著的优点: 首先, 不需要昂贵的过渡金属催 化剂; 其次, 参与反应的单体不需要有特殊的官能团. 而且由于这种反应所使用的催化剂具有很高的活性, 能 够参与反应的单体的范围就有了很大的扩展, 包括高电 子云密度和低电子云密度的单体, 酸性或碱性功能化单 体, 芳环、稠环或者杂环单体等. 不同单体的反应活性 不同, 反应过程中的交联程度有较大的差异, 最后得到
超交联聚合产物的孔结构以及性能也有很大不同. 氮气 吸附测试结果表明, 这一系列的超交联聚合物的 BET 比表面积从 636 到 $1421 \mathrm{~m}^{2} / \mathrm{g}$ 不等. 而不同聚合物网络 的不同性能具体表现如下：三苯基苯自聚的超交联聚合 物虽然没有最高的比表面积, 却具有最高的氢气吸附量 $0.0174 \mathrm{w}$. 出现这种现象的原因应该被归结为聚合物网 络中丰富的超微孔结构, 这种超微孔结构在小分子气体 的储存和分离中起到非常重要的作用. 对于二氧化碳的 吸附, 官能团的影响更为关键，含有碱性官能团的聚合 物具有更高的二氧化碳吸附热和吸附量. 在 $273 \mathrm{~K} / 1 \mathrm{bar}$ 条件下, 由吡咯和三苯基苯共聚的超交联聚合物显示出 最高达到 $0.204 w$ 的二氧化碳吸附量. 除此之外, 通过 小分子配体三苯基膦和三苯基苯的共聚，磷元素被引入 聚合物基体中用于络合钯纳米颗粒, 得到负载钯的微孔 材料对于催化 Suzuki-Miyaura 偶联反应显示出很好的催 化活性和选择性. 通过引入吡咯单体，三苯基苯超交联 a
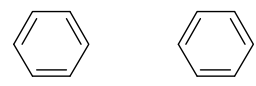

b

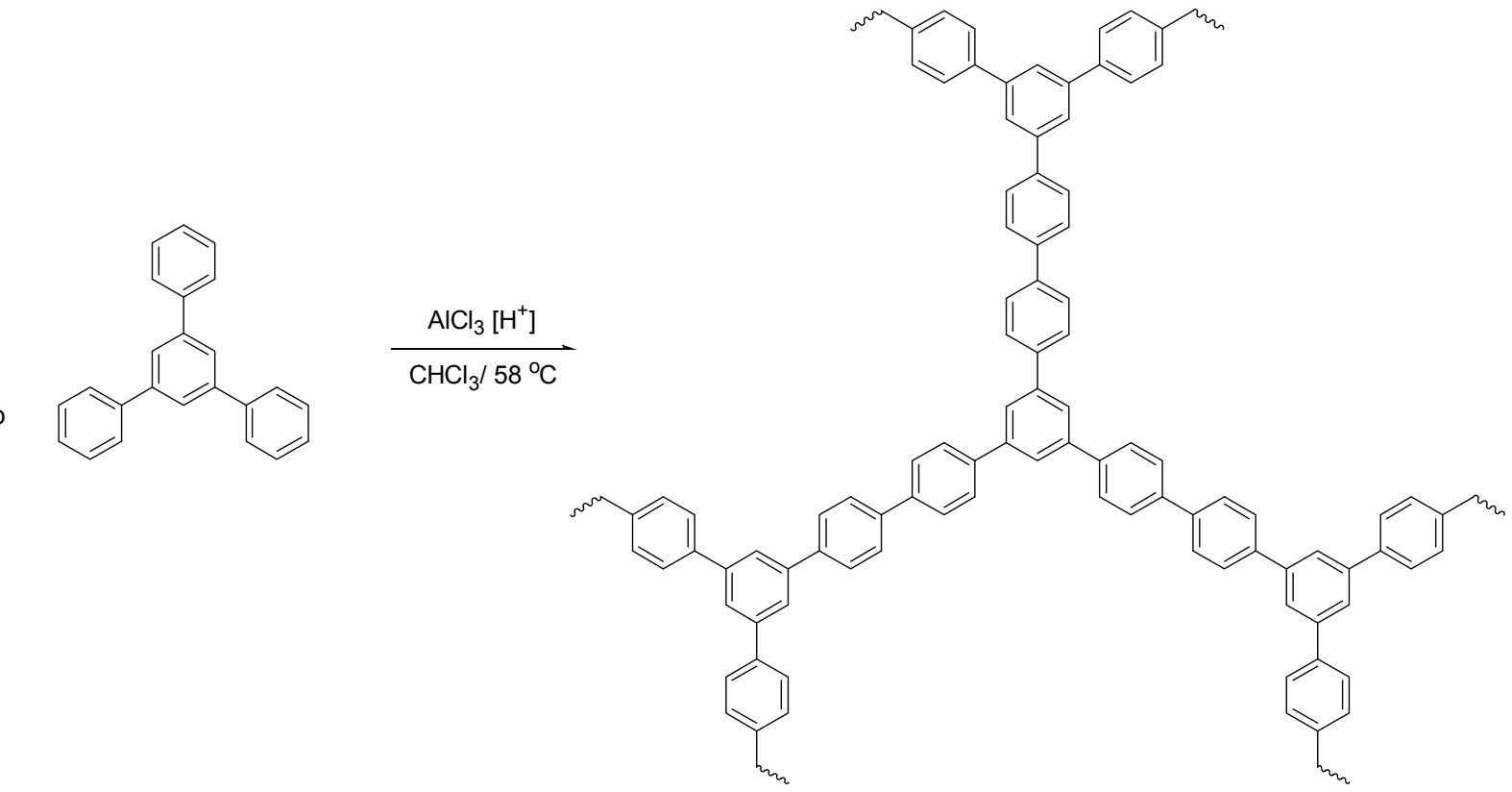

Lewis acid - Protic acid

c<smiles>c1cc2ccc3cccc4ccc(c1)c2c34</smiles><smiles></smiles><smiles>[R]c1ccccc1</smiles><smiles>c1ccc(-c2ccccn2)nc1</smiles>

图 5 (a) Scholl 偶联反应, (b)三苯基苯聚合物网络, (c)其它一些反应单体 ${ }^{[49]}$

Figure 5 (a) The typical Scholl reaction, (b) polymer networks form 1,3,5-triphenyl benzene, (c) other monomers ${ }^{[49]}$ 
聚合物能够显示出 $8.12 \times 10^{-6} \mathrm{~S} / \mathrm{m}$ 的电导率, 这一数值 远远高于大多数有机微孔材料. 而且至今为止, 这一电 导率在有机材料中仍然处于前列. 而且由于共轭结构的 存在, 这类聚合物能够显示出发光性能. 不同单体构建 的微孔聚合物网络分散在四氢呋喃中时, 在紫外灯的照 射下能够显示出多种不同的颜色(图 6).

(a)

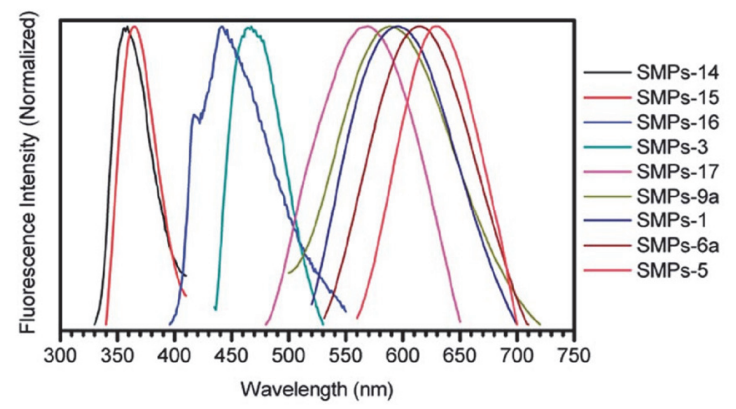

(b)

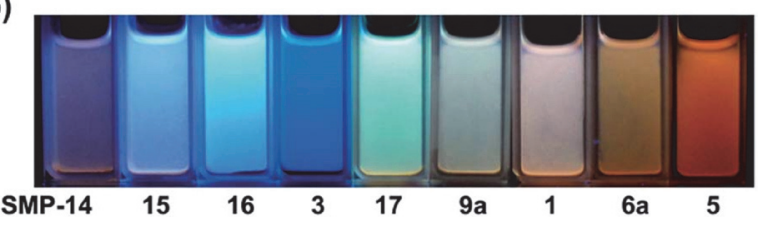

图 6 (a)超交联聚合物的荧光发射光谱, (b) 聚合物荧光照片 ${ }^{[49]}$

Figure 6 (a) Fluorescence emission spectra; (b) optical images under UV light at $365 \mathrm{~nm}^{[49]}$

同样的, Zhu 小组 ${ }^{[52]}$ 利用一些 3D 单体构建单元, 通 过 Scholl 偶联反应制备了一系列具有三维结构的超交联 聚合物. 聚合物最大比表面积为 $1119 \mathrm{~m}^{2} / \mathrm{g}$, 对甲烷和二 氧化碳吸附量分别为 $1.04 \mathrm{mmol} / \mathrm{g}$ 和 $3.52 \mathrm{mmol} / \mathrm{g}$, 显示 出在甲烷储存以及二氧化碳捕获方面的良好应用前景.

\section{4 外交联剂编织法}

虽然, 小分子自缩合方法扩展了单体选择范围, 然 而合成中所用到的构建单体单元必须含有可消除官能 团, 增加了微孔聚合物的制备成本; 而且, 目前普遍应 用的氯甲基官能团在反应过程中不可避免的会生成氯 化氢气体, 对生产设备以及环境会造成极大的危害. 如 果能从苯类等低官能度化合物制备得到高比表面积和 孔径可调控的超交联微孔聚合物, 不仅能够进一步扩大 单体选择范围, 而且消除了副产物的危害, 对于超交联 微孔聚合物材料的低成本大规模制备将是一个重大进 步.

2011 年, Tan 小组 ${ }^{[16]}$ 提出了一种新的合成策略, 即: 采用外交联剂二甲醇缩甲醛(formaldehyde dimethyl acetal, FDA)通过付-克反应 “编织” 低官能度刚性芳香族化 合物, 一步高效地合成高比表面积的微孔聚合物网络 (图 7), 氮气吸附测试结果表明, 这些超交联聚合物具有 丰富的微孔结构和较高的比表面积. 其中, 使用苯单体 制备的超交联聚合物具有最高的 BET 比表面积 1391 $\mathrm{m}^{2} / \mathrm{g}$. 不仅如此, 聚合物的比表面积、孔径和孔径分布 都能够通过调节不同单体和外交联剂之间的比例进行
调控. 而且聚合物网络的功能性能够通过引入不同的功 能性单体调节. 例如, 当氯苯和苯酚作为单体参与反应 时, 得到的聚合物网络中就含有了氯原子和羟基官能 才.
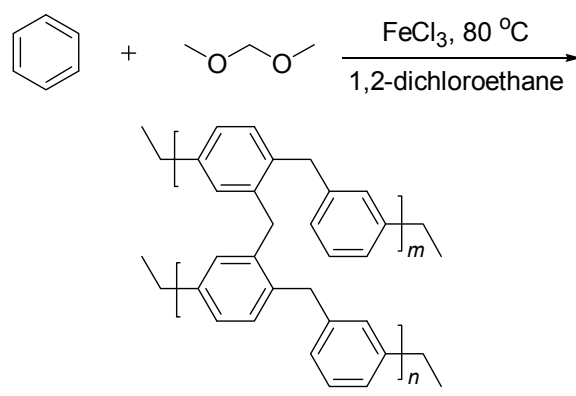

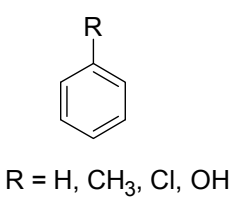

(a)

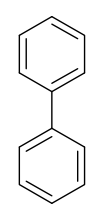

(b)

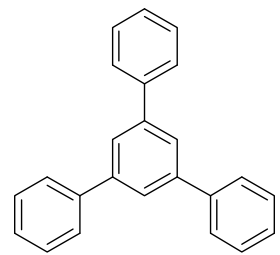

(c)
图 7 外交联方法合成超交联微孔聚合物 ${ }^{[16]}$

Figure 7 Using external crosslinker knitting aromatic compounds ${ }^{[16]}$

这种外交联的方法具有明显的优点: (1)构建单体单 元无需含有特定反应官能团，单体来源广泛; (2)合成条 件温和且原料廉价，可大规模生产; (3)得到聚合物网络 具有高比表面积和丰富微孔结构; (4)通过改变构建单元 可获得不同孔结构和功能化交联网络. 外交联方法为具 有独特微观形貌或者特殊性能超交联聚合物的合成提 供了一种简单有效的新思路，一经报道就受到国内外研 究学者的广泛关注.

Cooper 教授 ${ }^{[53]}$ 认为外交联剂 “编织” 法不仅极大扩 展了可选择单体的范围，同时采用低官能度芳香族化合 物又大大降低了生产的成本，在碳捕获材料的设计、合 成方面显示出极大的应用前景.

利用外交联剂 “编织” 法, Cooper 小组 ${ }^{[54]}$ 开展了一 系列工作. 他们通过苯胺与苯单体的共聚制备出了一系 列胺基含量不同的超交联网络. 通过调节苯胺与苯的单 体比例, 聚合物的比表面积能够被精确调控, 最高达到 $1100 \mathrm{~m}^{2} / \mathrm{g}$. 随着苯胺含量的提高, 超交联网络的二氧化 碳对氮气的吸附选择性可从 15.9 : 1 提高到最大值 $49.1: 1$.

他们 ${ }^{[55]}$ 还采用 “编织” 法以含有羟基的稠环为单体, 制备得到了含不同羟基官能度的超交联微孔聚合物，随 后研究了这类聚合物在水蒸气环境下的二氧化碳吸附 行为. 结果表明水蒸气的存在会使微孔材料吸附二氧化 碳的性能降低, 而增强聚合物网络的疏水性, 则可降低 这种影响.

最近, Copper 小组 ${ }^{[53]}$ 进一步研究了 “编织” 制备得 到的多种微孔材料在不同环境下(高温、高压)的二氧化 
碳吸附行为. 发现相比于更加刚性的金属有机骨架 (MOFs)、沸石咪唑酯骨架(ZIFs) 以及活性炭等材料, 这 种超交联编织微孔聚合物尽管比表面积并不突出, 高压 下却显示更高的二氧化碳吸附性能，原因可能在于这类 聚合物可以通过特殊的化学 “溶胀” 机理吸收更多的二 氧化碳. 同时这种超交联聚合物网络因为本身高疏水, 在高含量水蒸气气氛中仍然能够保持较高的二氧化碳 吸附量.

随后, 对于二氧化碳捕获材料的研究主要集中在合 成不同功能化的微孔聚合物. 国内外的研究学者设计、 使用了多种含不同官能团的单体构建单元, 通过外交联 “编织” 法制备了多种碳捕获材料.

$\mathrm{Zhu}$ 小组 ${ }^{[56]}$ 成功地使用了两种分别含有胺基和羟基 的四面体型单体通过外交联的方法制备出了高比表面 积的三维超交联网络. 相比于非功能化的聚合物材料, 含有官能团的网络显现出更高的二氧化碳吸附量和吸 附热.

Jiang 小组 ${ }^{[57]}$ 也报道了基于四苯乙烯和四苯乙烯二 醇两种单体共聚超交联聚合物的合成. 当聚合物完全由 四苯乙烯合成时, 比表面积最大可达到 $1980 \mathrm{~m}^{2} / \mathrm{g}$; 随着 聚合物中四苯乙烯二醇含量的提高, 聚合物比表面积二 氧化碳吸附量下降, 吸附选择性却随之提高. 当聚合物 完全由四苯乙烯二醇合成时, 虽然二氧化碳吸附量降低 到 $1.92 \mathrm{mmol} / \mathrm{g}$, 对二氧化碳选择性却达到最大值 119 .

随后, Jiang 小组 ${ }^{[58]}$ 选取含氮化合物四苯基联苯二胺 作为反应单体, 通过外交联的方法制备出高含氮量的超 交联聚合物前体. 虽然聚合物前体的比表面积只有 871 $\mathrm{m}^{2} / \mathrm{g}$, 经过氢氧化钾活化的碳化反应, 得到的碳材料比 表面积最终可达到 $2065 \mathrm{~m}^{2} / \mathrm{g}$. 由于具有较高的比表面 积和氮含量, 这种碳材料显示出高达 $6.51 \mathrm{mmol} / \mathrm{g}$ 的二 氧化碳吸附量, 被认为是一种理想的二氧化碳捕获材 料.

进一步的, Jiang 小组 ${ }^{[59]}$ 选取了多咔唑基苯作为反应 单体通过外交联 “编织” 法得到了比表面积高达 1845 $\mathrm{m}^{2} / \mathrm{g}$ 的微孔聚合物网络, 这种具有高含氮量的材料也显 示出高于一般材料的二氧化碳吸附性能和选择性.

$\mathrm{Zhu}$ 等 ${ }^{[60]}$ 通过外交联咔唑和 2,4,6-三咔唑基-1,3,5三嗪单体, 得到了两种含氮聚合物网络, 最高 BET 比表 面积可达 $913 \mathrm{~m}^{2} / \mathrm{g}$. 在 $273 \mathrm{~K} / 1 \mathrm{bar}$ 条件下, 这类含氮聚 合物网络表现出较高的二氧化碳吸附量 $0.18 \mathrm{w}$ 和可观 的吸附选择性 38 .

Han 小组 ${ }^{[61]}$ 同样选择了三种咔唑基的单体：1,4-二 (9-咔唑基)苯、4,4'-二(9-咔唑基)联苯和 1,3,5-三(9-咔唑 基)苯, 在外交联和氧化聚合反应共同作用下得到了最 高比表面积为 $1190 \mathrm{~m}^{2} / \mathrm{g}$ 的超交联微孔聚合物. 这类含 氮聚合物网络不仅显示出较高的二氧化碳吸附量, 而且 对甲苯和甲醛气体也有很好的吸附作用, 在净化空气方 面有着良好的潜在应用.
Saleh 等 ${ }^{[62]}$ 选取了多种含杂环的芳香族化合物作为 单体构建单元，通过 “编织” 法得到了一系列杂原子修 饰的微孔聚合物材料. 精确调控的孔径尺寸以及富含杂 原子的聚合物网络赋予这类材料高于一般材料的二氧 化碳吸附和选择性能.

外交联剂 “编织” 法被证明还能用于交联可再生的 生物质材料. Weber 小组 ${ }^{[63]}$ 选取了木质素作为反应物, 通过交联以及碳化得到了高比表面积的微孔碳材料. 这 种碳材料同样能够被用于二氧化碳的捕获.

除了用于制备具有二氧化碳吸附性能的功能化微 孔聚合物材料，外交联 “编织” 法在制备有机一无机复合 材料方面也显示出良好的应用前景.

Jiang 小组 ${ }^{[64]}$ 通过外交联的方法制备出一类新型的 有机硅复合材料. 分子中的苯环经过交联作用增强聚合 物网络的刚性, 微孔聚合物显示出高达 $1236 \mathrm{~m}^{2} / \mathrm{g}$ 的比 表面积. 并且由于聚合物网络中的硅原子对二氧化碳有 一定的亲和力, 这类材料能够显示出高于非功能化材料 的二氧化碳吸附量和吸附热.

Tan 小组 ${ }^{[65]}$ 分别采用外交联 “编织” 法以及 Scholl 偶联方法，以八苯基-POSS 为单体制备出了两种新型的 有机一无机复合材料. 氮气吸附测试结果表明这类材料 具有永久的微孔一介孔多级孔结构. 相比于同类的其他 材料，这类富含微孔结构的复合材料具有相当的氢气吸 附量和更高的二氧化碳吸附量, 且具有比纯有机材料更 高的热稳定性, 因而有潜力成为良好的二氧化碳捕获材 料.

外交联法还能够用于直接合成钯一磷络合的微孔聚 合物多相催化剂 ${ }^{[66]}$. 采用 “编织” 方法将苯和三苯基膦 共聚形成多孔有机材料, 络合二氯化钯后, 聚合物网络 的 BET 比表面积达到 $1036 \mathrm{~m}^{2} / \mathrm{g}$. 微孔结构的存在有效 地分散了钯活性位点, 阻止了钯的团聚和沉降, 使这种 催化体系展现出很高的催化活性, 在温和的反应条件 下，催化氯代芳烃与苯硼酸的偶联效果远高于均相的氯 化钯及二氯化二(三苯基膦)钯. 研究拓展了超交联微孔 聚合物材料在传统 Suzuki-Miyaura 偶联反应中的应用， 这类催化剂合成简单，催化条件温和，具有重要的应用 前景.

最近, Tan 小组 ${ }^{[67]}$ 制备出一种负载钯催化剂的 N-杂 化卡宾微孔聚合物网络. 通过 “编织” N-杂化卡宾类单 体与苯共聚, 得到微孔聚合物比表面积可达到 1229 $\mathrm{m}^{2} / \mathrm{g}$. 尽管钯的负载量很低，这类微孔聚合物材料同样 对多种卤代芳烃和苯硼酸的 Suzuki-Miyaura 偶联反应显 示出较高催化活性和选择性.

外交联剂 “编织” 方法还可用于芳杂环. Tan 小组 ${ }^{[68]}$ 选取了三种杂环单体：吡咯、噻吩和呋喃为构建单元, 分别通过单体自聚制备了孔壁修饰有大量杂原子的功 能化微孔聚合物网络(图 8). 聚合物的 BET 比表面积为 $437 \sim 726 \mathrm{~m}^{2} / \mathrm{g}$. 与类似的苯环交联形成的超交联微孔 
聚合物相比, 这类聚合物的比表面积明显降低. 但是, 杂环的引入使聚合物网络中具有了更多的杂原子孤对 电子, 通过偶极一偶极相互作用, 增加了对二氧化碳的 亲和力. 其中基于吡咯的交联网络显示出高达 117 的二 氧化碳对氮气的吸附选择性, 这在当时远远超过了其它 的微孔材料. 实验结果表明了引入杂环分子对二氧化碳 吸附量以及吸附选择性的巨大提升, 为碳捕获材料的发 展指明了新的方向.

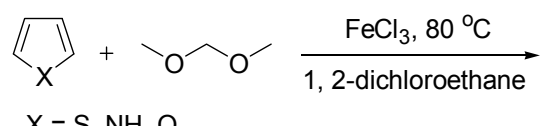

$$
\mathrm{X}=\mathrm{S}, \mathrm{NH}, \mathrm{O}
$$

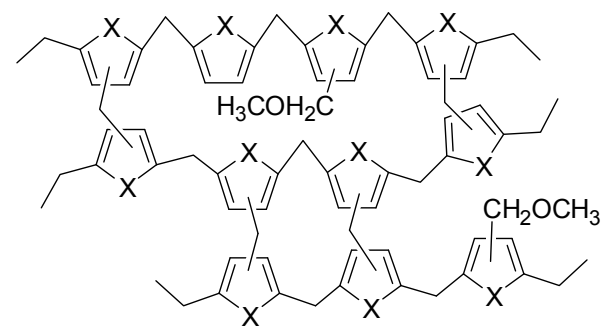

图 8 外交联方法合成杂环类超交联聚合物 ${ }^{[68]}$

Figure 8 The synthesis of aromatic heterocyclic microporous polymers $^{[68]}$

概括来说，使用这种简单高效的外交联方法能够通 过选择不同功能化的单体制备得到相应功能化的微孔 聚合物材料, 大大扩展了超交联聚合物的应用范围. 但 是, 对于微观形貌可控的超交联聚合物的报道还比较

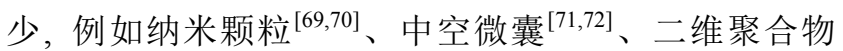
膜 ${ }^{[73,74]}$ 、整块材料 ${ }^{[44,75]}$ 等.
通过使用外交联的方法, Tan 小组 ${ }^{[69]}$ 成功的制备出 了一种高比表面积, 超顺磁的微孔聚合物纳米颗粒(图 9). 通过外交联聚苯乙烯壳层, 纳米颗粒的 BET 比表面 积可从无孔状态提高到 $560 \mathrm{~m}^{2} / \mathrm{g}$. 而被包裹聚苯乙烯之 后, 四氧化三铁纳米颗粒的饱和磁化强度仍然能够保留 三分之一，达到 $11.9 \mathrm{emu} \cdot \mathrm{g}^{-1}$. 这样富含微孔结构的磁 性纳米颗粒能够被用于有机污染物的吸附回收和磁性 药物输送系统.

最近, Tan 小组 ${ }^{[71]}$ 在中空微孔聚合物微囊的制备上 取得了一些进展. 首先, 通过一种简单的传统乳液聚合 将聚苯乙烯壳层包裹在二氧化硅纳米颗粒上. 随后通过 超交联反应并刻蚀二氧化硅核，得到了具有高比表面积 的有机微孔聚合物空心微囊(图 10). 通过调节乳液聚合 中单体苯乙烯和二乙烯基苯的比例, 得到的超交联聚合 物的比表面积和孔径尺寸可以被精确调控. 当二乙烯基 苯含量为苯乙烯 $0.005 \varphi$ 时, 得到的超交联聚合物具有 最高 BET 比表面积，随着二乙烯基苯含量的提高，比表 面积呈下降趋势. 中空微囊的空腔可以作为储存药物的 载体, 相比于实心的超交联纳米颗粒, 空腔大大提高了 纳米颗粒对于布洛芬的吸附量 $(62 \% \sim 68 \%)$. 在药物释 放阶段, 由于独特的孔结构和孔径尺寸, $0.1 \varphi$ 二乙烯基 苯的超交联微囊对布洛芬的释放显示出 0 级动力学曲 线，达到了对布洛芬的控制释放效果. 这种仅通过孔径 调节达到对药物控制释放的效果不受药物性质的影响, 对于所有药物的释放都能达到相似的结果, 因而在生物 医学方面有很广阔的应用前景.

除了在生物医学方面的应用, Tan 小组 ${ }^{[72]}$ 使用中空

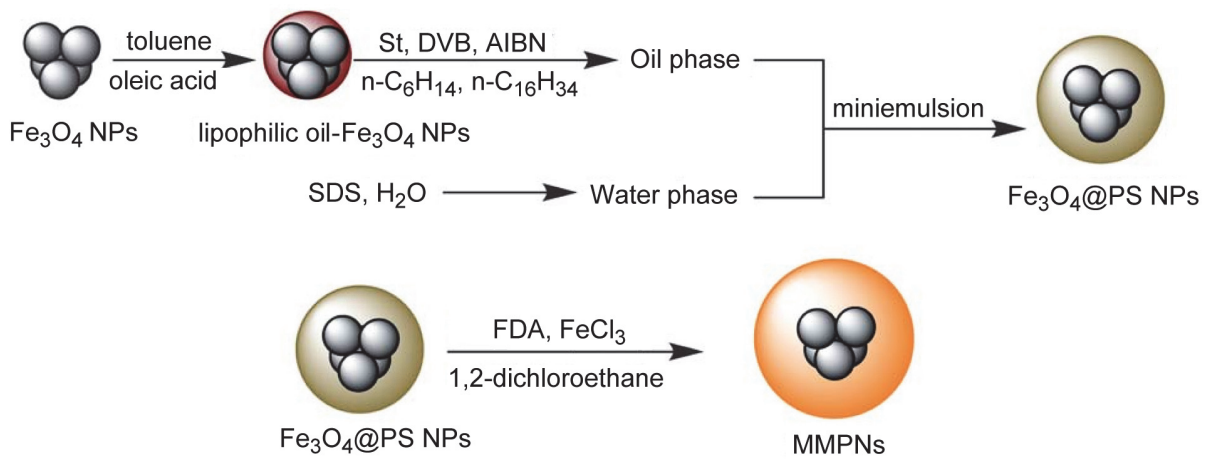

图 9 磁性微孔聚合物纳米颗粒的制备 ${ }^{[69]}$

Figure 9 The preparation of magnetic microporous polymer nanoparticles ${ }^{[69]}$

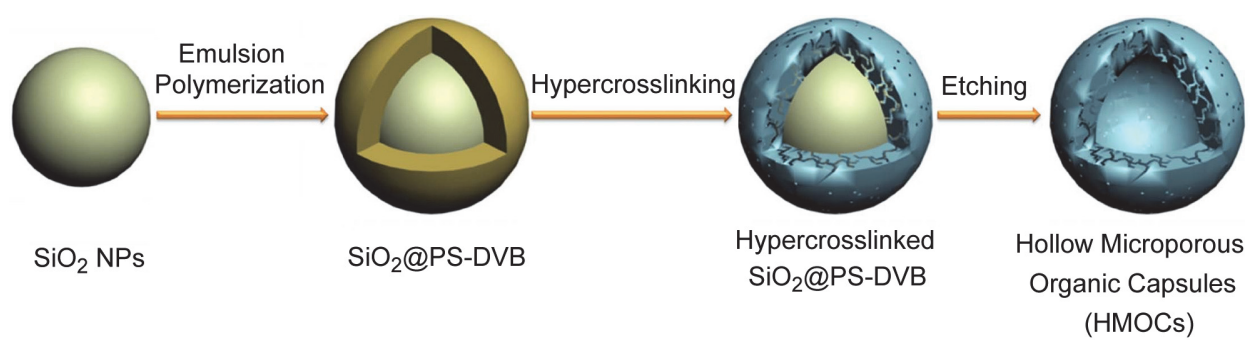

图 10 微孔聚合物中空微囊的制备 ${ }^{[71]}$

Figure 10 The preparation of hollow microporous organic capsules ${ }^{[71]}$ 
微囊作为纳米反应器制备了负载铂金属的聚合物纳米 颗粒. 聚合物的微孔结构保证了铂颗粒的高度分散, 这 种负载铂的微孔聚合物微囊在硝基苯的还原反应中显 示出了较高的催化活性和选择性.

Shi 等 ${ }^{[76]}$ 设计并合成了一种核壳结构的微孔聚合物 网络，在其空腔原位还原得到金纳米颗粒后作为高效的 纳米反应器用于催化降解环己基过氧化氢. 金纳米颗粒 被空腔隔开最大限度了减少了团聚, 因而能够保持较高 的催化活性和选择性.

相比于三维中空微囊结构模型, 具有二维微观形貌 的多孔膜在能源环境方面对小分子的分离有很大的优 势. Qiao 等 ${ }^{[74]}$ 以无孔聚苯乙烯膜前体为模版, 通过同位 的外交联反应制备出一种高渗透性和高选择性聚合物 分子笁膜(图 11). 通过 AFM、SEM、HR-TEM 对聚合物 膜的形貌研究发现, 这种超交联聚合物膜显示出夹层的 多级孔结构，包括了聚合物纳米颗粒覆盖的介孔表面、 中空的大孔内层以及具有致密微孔结构的中间层. 这种 结构的形成是由于聚合物分子链扩散和交联反应的共 同作用: 首先, 聚苯乙烯膜表面的分子与交联剂快速反 应生成了微孔结构的聚合物表层; 交联剂和催化剂通过 表层的微孔结构扩散进入聚苯乙烯膜内部进一步反应 加深了微孔层的厚度, 同时内层聚苯乙烯分子的向外扩 散, 向外扩散的聚苯乙烯分子同时在聚合物膜微孔层表 面与交联剂发生反应, 形成了介孔纳米颗粒外层; 而聚 苯乙烯分子的向外扩散导致了聚苯乙烯膜内部的空缺, 最终形成了中空的大孔内层结构. 氮气吸附测试分析显 示出这类微孔聚合物膜具有夹杂着轻度滞后环类似 I 型 的吸附等温线, 证明了微孔、介孔结构的存在. 超交联 聚合物的比表面积主要受微孔含量的影响, 交联时间越 长，微孔层的厚度越大，聚合物比表面积也越高. 当交 联时间为 $6 \mathrm{~h}$, 得到聚合物的比表面积达到最大 618 $\mathrm{m}^{2} / \mathrm{g}$, 之后再延长交联时间, 聚合物比表面积并没有明 显变化. 对这类微孔聚合物分子篮膜进行气体选择性测 试发现, 随着交联时间的延长, 二氧化碳的渗透率逐渐 增大, 但是二氧化碳对氮气的选择性略微降低. 当二氧 化碳渗透率为 5561 时, 二氧化碳对氮气的选择性仍然 达到 18.5, 这一结果能够和许多商业化气体分离膜相比 较. 这类通过外交联 “编织” 法制备得到的微孔聚合物 分子篎膜合成方法简单、原料廉价, 存在大规模生产的 可能，因而在气体分离应用方面极具发展潜力.

具有多级孔形貌结构的整块材料，是一种能够连接 微观尺度和宏观尺度的特殊材料. 整块材料内部具有连 通的大孔、介孔和微孔结构为质量能量的快速传递提供 了物质基础, 从而引起了广泛的研究关注 ${ }^{[77]}$. Maya 等 ${ }^{[75]}$ 报道了一种通过外交联方法制备超交联苯乙烯类多级 孔聚合物整块材料的方法, 并探究了不同反应条件以及 不同的交联剂对得到聚合物孔结构和性能的影响. 使用 BCMBP 作为交联剂得到的整块材料的比表面积达到最

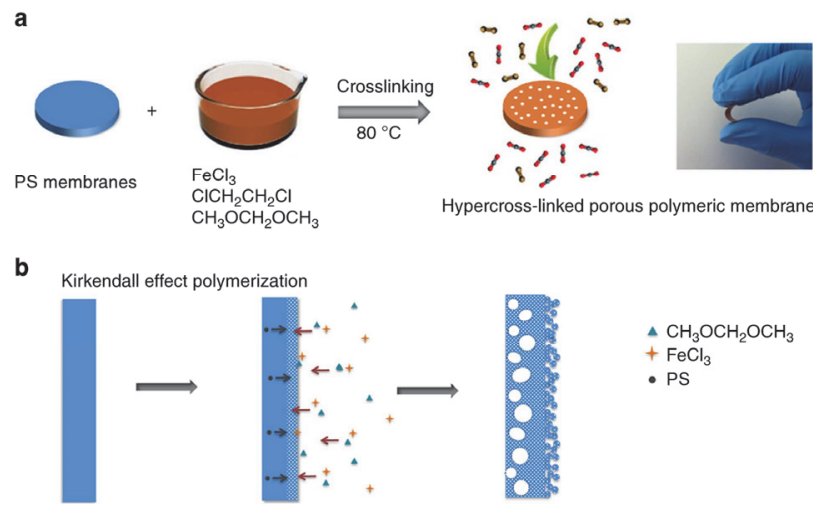

图 11 (a)超交联微孔聚苯乙烯膜的合成, (b)多级孔结构形成机理 ${ }^{[74]}$ Figure 11 (a) The synthesis of hypercrosslinked microporous polystyrene membranes, (b) the mechanism for the formation of hierarchical porous structure ${ }^{[74]}$

大 $900 \mathrm{~m}^{2} / \mathrm{g}$. 由于具有较高比表面积和内部连通的孔结 构，这种超交联的整块材料可以作为液相色谱固定相用 于有机小分子的反相分离.

除了制备有机微孔聚合物材料，超交联的方法还可 以被用于合成由微孔聚合物衍生出来的其它材料, 例如 具有多级孔结构的碳材料 ${ }^{[34,47,78,79]} . \mathrm{Wu}$ 小组 ${ }^{[79]}$ 通过交联 单分散的聚苯乙烯纳米微球得到了具有多级孔结构的 超交联微球。交联作用提高了聚合物网络的刚性，保持 了碳化后纳米微球的形貌. 之后, $\mathrm{Wu}$ 小组 ${ }^{[78]}$ 将研究进 一步加深, 使用了更大粒径的聚苯乙烯纳米微球进行超 交联反应. 微球表面的聚苯乙烯能够被交联形成保护 层，减少微球之间的交联作用，从而减少了由于微球之 间堆积形成的介孔和大孔结构. 碳化后的微球仍然保持 了单分散性，在吸附、分离、药物负载、催化等领域展 现出极大的应用前景.

综上所述：由于温和的反应条件、广泛的反应单体 来源，这种交联剂 “编织” 法已经发展成为了一种非常 实用的普适方法，不仅能被用于合成微孔聚合物材料， 而且在其它一些材料的合成中起到越来越重要的作用.

\section{5 总结与展望}

近几十年，超交联微孔聚合物经历了非常快速的发 展，不论合成方法还是应用范围都有了极大的扩展. 相 比于 PIMs、CMPs 和 COFs 等有机微孔聚合物，超交联 聚合物有着明显的优点: 良好的热稳定、物理化学稳定 性、温和的合成条件、来源广泛的反应单体和廉价的催 化剂等. 这些优势为超交联聚合物的工业化生产提供了 良好的可行性基础.

根据合成方法的差异, 超交联聚合物主要可以通过 以下三种方法制备得到：(1)含官能团聚合物前体后交 联. (2)功能化小分子一步法自缩聚. (3)外交联剂 “编织” 芳香族单体. 
最初的超交联聚合物 Davankov 树脂是通过聚苯乙 烯类聚合物前体的后交联得到的，这类聚合物的比表面 积通常都比较高, 被用于柱色谱固定相中分离有机小分 子. 之后, Cooper 小组采用功能化的氯化茮基芳环化合 物的缩合反应同样得到了高比表面积的超交联网络. 但 是，这一类反应要求单体中必须含有可消除的官能团， 因而限制了能够参与反应单体选择范围. 2011 年, Tan 小 组取得了突破性的进展, 采用外交联剂通 “编织” 刚性 的芳环结构单元得到了一系列高比表面积的新型超交 联网络. 相比于之前的方法, 外交联方法有非常明显的 优点: (1)单体无需含有特定官能团, 来源广泛; (2)合成 条件温和且原料廉价, 可大规模生产; (3)得到聚合物网 络具有高比表面积和丰富微孔结构; (4)通过改变构建单 元可获得不同孔结构和功能化聚合物网络.

由于其独特的优势, 超交联聚合物在气体储存、分 离、吸附以及多相催化等领域展现出广泛的潜在应用. 尽管如此, 超交联聚合物的发展仍然面临许多挑战. 例 如，超交联反应的最大缺点是交联反应发生的随机性， 得到的聚合物网络通常没有规整的结构, 这就大大限制 了超交联聚合物材料的实际应用. 未来超交联微孔聚合 物的发展很有可能包括以下几个方向: (1)寻找新的合成 方法来制备更高比表面积的超交联聚合物; (2)探索能够 得到规整结构的可控合成方法; (3)制备多功能化超交联 聚合物并扩展其在光电、传感器、半导体器件等领域的 应用。

\section{作者简介}

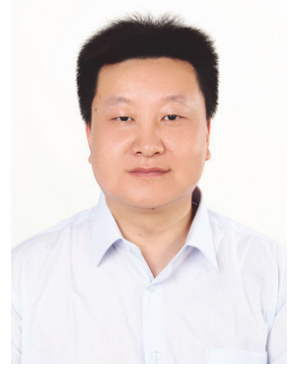

谭必恩, 华中科技大学化学与化工学院教授、博士生导师, 材料化学与服役失效湖北省重点实验室主任, 湖北省 “楚天学 者” 特聘教授. 湖北省杰出青年人才基金获得者, 教育部“新 世纪优秀人才支持计划” 入选者. 同时兼任全国青联委员、湖 北省政协委员等社会职务. 1989 年至 1996 年在湖北大学化学 系学习, 先后获学士、理学硕士学位. 1996 年进入华南理工大 学材料学院攻读博士学位, 与中国运载火箭技术研究院航天 材料及工艺研究所合作开展博士论文研究, 1999 年获工学博 士学位. 1999 年至 2001 年在北京航空材料研究院先进复合材 料国防重点实验室从事博士后研究. 2001 年至 2007 年在英国 利物浦大学任 Research Associate. 2007 年 9 月起在华中科技大 学化学系工作. 先后参与和承担了国家自然科学基金项目、湖 北省青年杰出人才基金、国防重点预研项目、中国博士后基金 项目以及英国工程和自然科学研究委员会(EPSRC)等多项课 题. 目前主要研究兴趣集中于纳米材料、微孔聚合物合成及气
体储存研究、超临界流体化学、空间有机材料、特种高分子材 料及树脂基先进复合材料等领域.

谭良骁, 2012 年本科毕业于华中科技大学化学与化工学 院, 同年加入谭必恩教授课题组攻读博士学位. 主要研究方向 为新型超交联微孔聚合物的合成及应用.

\section{References}

[1] Sing, K. S. W.; Everett, D. H.; Haul, R. A. W.; Moscou, L.; Pierotti, R. A.; Pouquerol, J.; Siemieniewska, T. Pure Appl. Chem. 1985, 57, 603.

[2] Wu, D.; Xu, F.; Sun, B.; Fu, R.; He, H.; Matyjaszewski, K. Chem. Rev. 2012, 112, 3959.

[3] Xing, W.; Zhuo, S.-P.; Gao, X.-L.; Huang, C.-C. Acta Chim. Sinica 2009, 67, 1430. (邢伟, 禚淑萍, 高秀丽, 黄丛聪, 化学学报, 2009, 67, 1430.)

[4] Mui, E. L. K.; Ko, D. C. K.; McKay, G. Carbon 2004, 42, 2789.

[5] Roth, W. J.; Nachtigall, P.; Morris, R. E.; Čejka, J. Chem. Rev. 2014, 114, 4807.

[6] Ciriminna, R.; Fidalgo, A.; Pandarus, V.; Béland, F.; Ilharco, L. M. Pagliaro, M. Chem. Rev. 2013, 113, 6592.

[7] Dawson, R.; Cooper, A. I.; Adams, D. J. Prog. Polym. Sci. 2012, 37, 530.

[8] Pandey, P.; Katsoulidis, A. P.; Eryazici, I.; Wu, Y.; Kanatzidis, M. G.; Nguyen, S. T. Chem. Mater. 2010, 22, 4974.

[9] Weber, J.; Antonietti, M.; Thomas, A. Macromolecules 2008, 41, 2880 .

[10] Wang, H.-X. Petrochemical Technology 2002, 6, 483. (王红霞, 石 油化工, 2002, 6, 483.)

[11] Cooper, A. I. Adv. Mater. 2009, 21, 1291.

[12] Jiang, J.; Li, Y.; Wu, X.; Xiao, J.; Adams, D. J.; Cooper, A. I. Macromolecules 2013, 46, 8779.

[13] McKeown, N. B.; Budd, P. M. Chem. Soc. Rev. 2006, 35, 675.

[14] El-Kaderi, H. M.; Hunt, J. R.; Mendoza-Cortes, J. L.; Cote, A. P.; Taylor, R. E.; O'Keeffe, M.; Yaghi, O. M. Science 2007, 316, 268.

[15] Xu, S.; Luo, Y.; Tan, B. Macromol. Rapid Commun. 2013, 34, 471.

[16] Li, B.; Gong, R.; Wang, W.; Huang, X.; Zhang, W.; Li, H.; Hu, C.; Tan, B. Macromolecules 2011, 44, 2410.

[17] Wood, C. D.; Tan, B.; Trewin, A.; Niu, H.; Bradshaw, D.; Rosseinsky, M. J.; Khimyak, Y. Z.; Campbell, N. L.; Kirk, R.; Stöckel, E.; Cooper, A. I. Chem. Mater. 2007, 19, 2034.

[18] Yuan, S.; Dorney, B.; White, D.; Kirklin, S.; Zapol, P.; Yu, L.; Liu, D. Chem. Commun. 2010, 46, 4547.

[19] Holst, J. R.; Stöckel, E.; Adams, D. J.; Cooper, A. I. Macromolecules 2010, 43, 8531 .

[20] Pandey, P.; Farha, O. K.; Spokoyny, A. M.; Mirkin, C. A.; Kanatzidis, M. G.; Hupp, J. T.; Nguyen, S. T. J. Mater. Chem. 2011, 21, 1700

[21] Chen, Q.; Luo, M.; Hammershøj, P.; Zhou, D.; Han, Y.; Laursen, B. W.; Yan, C.; Han, B. J. Am. Chem. Soc. 2012, 134, 6084

[22] Schmidt, J.; Weber, J.; Epping, J. D.; Antonietti, M.; Thomas, A. Adv. Mater. 2009, 21, 702.

[23] Davankov, V. A.; Rogozhin, S. V.; Tsyurupa, M. P. US 3729457, 1971. [Chem. Abstr. 1971, 75, 6841]

[24] Jiang, J.-X.; Cooper, A. In Functional Metal-Organic Frameworks: Gas Storage, Separation and Catalysis, Vol. 293, Ed.: Schröder, M. Springer, Berlin Heidelberg, 2010, Chapter 1.

[25] Tsyurupa, M. P.; Davankov, V. A. React. Funct. Polym. 2006, 66, 768.

[26] Germain, J.; Fréchet, J. M. J.; Svec, F. J. Mater. Chem. 2007, 17, 4989.

[27] Davankov, V. A.; Ilyin, M. M.; Tsyurupa, M. P.; Timofeeva, G. I.; Dubrovina, L. V. Macromolecules 1996, 29, 8398.

[28] Hradil, J.; Králová, E. Polymer 1998, 39, 6041.

[29] Veverka, P.; Jeřábek, K. React. Funct. Polym. 1999, 41, 21.

[30] Ahn, J.-H.; Jang, J.-E.; Oh, C.-G.; Ihm, S.-K.; Cortez, J.; Sherrington, D. C. Macromolecules 2006, 39, 627.

[31] Li, B.; Gong, R.; Luo, Y.; Tan, B. Soft Matter 2011, 7, 10910.

[32] Abbott, L. J.; Colina, C. M. Macromolecules 2014, 47, 5409.

[33] Seo, M.; Kim, S.; Oh, J.; Kim, S.-J.; Hillmyer, M. A. J. Am. Chem. Soc. 2015, 137, 600 .

[34] Li, Z.; Wu, D.; Huang, X.; Ma, J.; Liu, H.; Liang, Y.; Fu, R.; Matyjaszewski, K. Energy Environ. Sci. 2014, 7, 3006. 
[35] Davankov, V.; Tsyurupa, M.; Ilyin, M.; Pavlova, L. J. Chromatogr. A 2002, 965, 65 .

[36] Tsyurupa, M. P.; Davankov, V. A. React. Funct. Polym. 2002, 53, 193.

[37] Wood, C. D.; Tan, B.; Trewin, A.; Su, F.; Rosseinsky, M. J.; Bradshaw, D.; Sun, Y.; Zhou, L.; Cooper, A. I. Adv. Mater. 2008, 20, 1916.

[38] Nielsen, C. J.; Herrmann, H.; Weller, C. Chem. Soc. Rev. 2012, 41, 6684.

[39] Martín, C. F.; Stöckel, E.; Clowes, R.; Adams, D. J.; Cooper, A. I.; Pis, J. J.; Rubiera, F.; Pevida, C. J. Mater. Chem. 2011, 21, 5475.

[40] Farha, O. K.; Spokoyny, A. M.; Hauser, B. G.; Bae, Y.-S.; Brown, S. E.; Snurr, R. Q.; Mirkin, C. A.; Hupp, J. T. Chem. Mater. 2009, 21, 3033.

[41] Chen, S.; Zhang, J.; Wu, T.; Feng, P.; Bu, X. J. Am. Chem. Soc. 2009, 131, 16027.

[42] Couck, S.; Denayer, J. F. M.; Baron, G. V.; Rémy, T.; Gascon, J.; Kapteijn, F. J. Am. Chem. Soc. 2009, 131, 6326.

[43] Yang, Y.; Zhang, Q.; Zhang, S.; Li, S. Polymer 2013, 54, 5698.

[44] Yang, Y.; Zhang, Q.; Zhang, S.; Li, S. RSC Adv. 2014, 4, 5568.

[45] Chaikittisilp, W.; Kubo, M.; Moteki, T.; Sugawara-Narutaki, A.; Shimojima, A.; Okubo, T. J. Am. Chem. Soc. 2011, 133, 13832.

[46] Wu, Y.; Wang, D.; Li, L.; Yang, W.; Feng, S.; Liu, H. J. Mater. Chem. A 2014, 2, 2160.

[47] Li, Z.; Wu, D.; Liang, Y.; Fu, R.; Matyjaszewski, K. J. Am. Chem. Soc. 2014, 136, 4805.

[48] Luo, Y.; Zhang, S.; Ma, Y.; Wang, W.; Tan, B. Polym. Chem. 2013, 4, 1126.

[49] Li, B.; Guan, Z.; Yang, X.; Wang, W. D.; Wang, W.; Hussain, I.; Song, K.; Tan, B.; Li, T. J. Mater. Chem. A 2014, 2, 11930.

[50] Tan, B.-E. Sino-Deutsch Symposium GZ842 on pi - Structured Polymers and Carbon Nanomaterials for Energy Applications, Beijing, 2012, p. 44.

[51] Grzybowski, M.; Skonieczny, K.; Butenschön, H.; Gryko, D. T. Angew. Chem. Int. Ed. 2013, 52, 9900.

[52] Li, L.; Ren, H.; Yuan, Y.; Yu, G.; Zhu, G. J. Mater. Chem. A 2014, 2,11091 .

[53] Woodward, R. T.; Stevens, L. A.; Dawson, R.; Vijayaraghavan, M.; Hasell, T.; Silverwood, I. P.; Ewing, A. V.; Ratvijitvech, T.; Exley, J. D.; Chong, S. Y.; Blanc, F.; Adams, D. J.; Kazarian, S. G.; Snape, C. E.; Drage, T. C.; Cooper, A. I. J. Am. Chem. Soc. 2014, 136, 9028.

[54] Dawson, R.; Ratvijitvech, T.; Corker, M.; Laybourn, A.; Khimyak, Y. Z.; Cooper, A. I.; Adams, D. J. Polym. Chem. 2012, 3, 2034.

[55] Dawson, R.; Stevens, L. A.; Drage, T. C.; Snape, C. E.; Smith, M. W.; Adams, D. J.; Cooper, A. I. J. Am. Chem. Soc. 2012, 134, 10741 .
[56] Jing, X.; Zou, D.; Cui, P.; Ren, H.; Zhu, G. J. Mater. Chem. A 2013, $1,13926$.

[57] Yao, S.; Yang, X.; Yu, M.; Zhang, Y.; Jiang, J.-X. J. Mater. Chem. A 2014, 2, 8054

[58] Yang, X.; Yu, M.; Zhao, Y.; Zhang, C.; Wang, X.; Jiang, J.-X. J. Mater. Chem. A 2014, 2, 15139.

[59] Yang, X.; Yu, M.; Zhao, Y.; Zhang, C.; Wang, X.; Jiang, J.-X. RSC $A d v .2014,4,61051$.

[60] Zhu, X.; Mahurin, S. M.; An, S.-H.; Do-Thanh, C.-L.; Tian, C.; Li, Y.; Gill, L. W.; Hagaman, E. W.; Bian, Z.; Zhou, J.-H.; Hu, J.; Liu, H.; Dai, S. Chem. Commun. 2014, 50, 7933.

[61] Zhu, J.-H.; Chen, Q.; Sui, Z.-Y.; Pan, L.; Yu, J.; Han, B.-H. J. Mater. Chem. A 2014, 2, 16181.

[62] Saleh, M.; Lee, H. M.; Kemp, K. C.; Kim, K. S. ACS Appl. Mater. Interfaces 2014, 6, 7325 .

[63] Meng, Q. B.; Weber, J. ChemSusChem 2014, 7, 3312.

[64] Zhang, Y.; Li, Y.; Wang, F.; Zhao, Y.; Zhang, C.; Wang, X.; Jiang, J.-X. Polymer 2014, 55, 5746 .

[65] Wang, S.; Tan, L.; Zhang, C.; Hussain, I.; Tan, B. J. Mater. Chem. A 2015, 3,6542.

[66] Li, B.; Guan, Z.; Wang, W.; Yang, X.; Hu, J.; Tan, B.; Li, T. Adv. Mater. 2012, 24, 3390.

[67] Xu, S.; Song, K.; Li, T.; Tan, B. J. Mater. Chem. A 2015, 3, 1272.

[68] Luo, Y.; Li, B.; Wang, W.; Wu, K.; Tan, B. Adv. Mater. 2012, 24, 5703.

[69] Yang, X.; Li, B.; Majeed, I.; Liang, L.; Long, X.; Tan, B. Polym. Chem. 2013, 4, 1425.

[70] Li, B.; Huang, X.; Gong, R.; Ma, M.; Yang, X.; Liang, L.; Tan, B. Int. J. Hydrogen Energy 2012, 37, 12813.

[71] Li, B.; Yang, X.; Xia, L.; Majeed, M. I.; Tan, B. Sci. Rep. 2013, 3 , 2128

[72] Yang, X.; Song, K.; Tan, L.; Hussain, I.; Li, T.; Tan, B. Macromol. Chem. Phys. 2014, 215, 1257.

[73] Huang, X.-W.; Deng, J.-Y.; Xu, L.; Shen, P.; Zhao, B.; Tan, S.-T Acta Chim. Sinica 2012, 70, 1604. (黄先威, 邓继勇, 许律, 沈平, 赵斌, 谭松庭, 化学学报, 2012, 70, 1604.)

[74] Qiao, Z.; Chai, S.; Nelson, K.; Bi, Z.; Chen, J.; Mahurin, S. M.; Zhu, X.; Dai, S. Nat. Commun. 2014, 5, 3705.

[75] Maya, F.; Svec, F. Polymer 2014, 55, 340.

[76] Shi, S.; Chen, C.; Wang, M.; Ma, J.; Ma, H.; Xu, J. Chem. Commun. 2014, 50, 9079.

[77] Namera, A.; Nakamoto, A.; Saito, T.; Miyazaki, S. J. Sep. Sci. 2011, 34, 901.

[78] Ouyang, Y.; Shi, H.; Fu, R.; Wu, D. Sci. Rep. 2013, 3, 1430.

[79] Zeng, Q.; Wu, D.; Zou, C.; Xu, F.; Fu, R.; Li, Z.; Liang, Y.; Su, D. Chem. Commun. 2010, 46, 5927. 